\title{
La correspondance de Georges Lefebvre avec Robert R. Palmer (1948-1959)
}

\section{James Friguglietti}

\section{(2) OpenEdition \\ 1 Journals}

\section{Édition électronique}

URL : https://journals.openedition.org/ahrf/11538

DOI : $10.4000 /$ ahrf.11538

ISSN : 1952-403X

Éditeur :

Armand Colin, Société des études robespierristes

\section{Édition imprimée}

Date de publication : 1 octobre 2009

Pagination : 93-132

ISBN : 978-2-200-92560-4

ISSN : 0003-4436

\section{Référence électronique}

James Friguglietti, «La correspondance de Georges Lefebvre avec Robert R. Palmer (1948-1959) », Annales historiques de la Révolution française [En ligne], 358 | octobre-décembre 2009, mis en ligne le 01 octobre 2012, consulté le 23 avril 2022. URL : http://journals.openedition.org/ahrf/11538; DOI : https://doi.org/10.4000/ahrf.11538 


\title{
LA CORRESPONDANCE DE GEORGES LEFEBVRE AVEC L'HISTORIEN GORDON H. McNEIL (1940-1954)
}

\author{
James FRIGUGLIETTI
}

C'est grâce à la générosité de M. David McNeil que l'éditeur a pu obtenir les huit lettres adressées à son père Gordon $\mathrm{H}$. McNeil par Georges Lefebvre entre 1940 et 1954. Né à Ottawa (Canada) en 1913, McNeil a fait ses études supérieures à l'Université de Chicago. Sous la direction de Louis Gottschalk, il rédigea un travail de maîtrise sur la panthéonisation de Jean-Jacques Rousseau (1937), puis sa thèse de doctorat sur le culte de Rousseau et l'esprit révolutionnaire en France de 1750 à 1800 (1941). McNeil passa l'année scolaire 1938-1939 à Paris et à Genève, où il faisait des recherches. Entre-temps il suivit un cours de Charles Seignobos à la Sorbonne ${ }^{1}$. C'est sans doute à ce moment-là que McNeil fit la connaissance de Lefebvre qui lui donna des conseils pour sa thèse. Rentré aux États-Unis à la veille de la guerre, il acheva sa thèse de doctorat et prépara aussi une esquisse biographique de Charles Seignobos, incorporant des renseignements que celui-ci lui fournissait par l'intermédiaire de Lefebvre $^{2}$.

Interrompus par le conflit, leurs contacts devaient reprendre en 1945. Bien que McNeil ne contribua pas aux Annales historiques de la Révolution française, il adhéra à la Société des études robespierristes dès sa reconstitution en $1945^{3}$ et Lefebvre signala aux lecteurs de la revue la publication de son article sur le culte de Rousseau sous la Révolution,

(1) Gordon H. McNeil, «Charles Seignobos (1854-1942)», dans S. William Halperin (éd.), Essays in Modern European Historiography, Chicago et Londres, University of Chicago Press, 1970, p. 355.

(2) Ibid., p. 352-369.

(3) « Membres de la Société des études robespierristes », AHRF, t. 18, 1946, p. 398. 
paru la même année ${ }^{4}$. En retour, McNeil lui expédiait des colis de nourriture qui étaient fort appréciés.

La guerre finie, il poursuivit sa carrière académique, d'abord à Coe College, ensuite à l'Université d'Arkansas dès 1953. Vingt ans après avoir pris sa retraite, McNeil s'est éteint le 20 mai 2001.

Après la disparition de Lefebvre en 1959, il rédigea une étude détaillée sur la carrière de son ami français ${ }^{5}$. Heureusement McNeil conserva les lettres ci-dessous, qui fournissent des détails inédits sur l'œuvre ainsi que sur la vie privée de Lefebvre.

James FrigugLIETTI

Montana State University-Billings

Billings, Montana 59101

États-Unis

jfriguglietti@msubillings.edu

(4) « Revue de la presse », ibid., p. 191. « Le culte de Rousseau et la Révolution française », article paru dans The Journal of the History of Ideas, t. 6, avril 1945, p. 197-212.

(5) Gordon H. McNeIL, « Georges Lefebvre (1974-1959) », dans S. William Halperin (éd.), op. cit., p. 160-174. 
Université de Paris

Paris, le 5 juin 1940

Faculté des lettres

Institut d'Histoire

de la Révolution Française

Cher Monsieur McNeil,

J'ai dû attendre quelque temps pour vous répondre. J'avais bien souvenir d'un article dans « Le Temps » sur [Charles] Seignebos, mais je n'en avais pas la date, et l'auteur, Pierre Mille ${ }^{1}$, un de nos meilleurs journalistes, m'a répondu qu'il ne l'avait non plus et que, dans les circonstances actuelles, il ne fallait pas penser à faire la recherche. J'ai donc écrit à Seignobos lui-même et il m'a envoyé une notice autobiographique dont je vous envoie copie, ainsi que d'un passage de la lettre de Pierre Mille qui, je pense, vous intéressera.

Sur la méthode de Seignebos, vous vous reporterez à l'Introduction aux études historiques qu'il a publiée avec Ch[arles] V[ictor] Langlois ${ }^{2}$ et qui est bien connu aussi qu'aux Études de politique et d'histoire qu'on a éditées pour lui rendre hommage.J'espère que vous trouverez ces ouvrages en Amérique.

J'ai été content de recevoir de vos nouvelles et de vous savoir établi dans une université. J'ignorais ce que vous étiez devenu et je m'en trouvais inquiet. En partant, vous ne m'avez pas laissé d'adresse, en sorte que je n'ai pu vous envoyer les Annales [historiques de la Révolution française]. Veuillez bien me dire quel est le premier des numéros qui vous manquent et je vous les expédierai : nous avons réussi à continuer la publication. Le second numéro de 1940 (mars-avril) a paru et nous préparons celui de mai-juin. Il a fallu diminuer le nombre de pages et l'on vient de nous ordonner de le réduire encore. Le papier se fait rare ici.

Je ne vous parle pas longuement de la situation générale car vous la connaissez aussi bien que moi. Nous avons été bombardé avant-hier et les morts sont plus nombreux qu'on n'avait cru d'abord : près de 250. D'autre part, nous allons être attaqués par l'Italie. Nous tiendrons, je pense, jusqu'à la mort. Mais la situation est périlleuse. Quand vous recevrez cette lettre, la poussée Allemande sur Paris sera sans doute en cours.

Il me semble que les Américains ne peuvent manquer de faire quelques réflexions sur l'état des choses. Si nous succombons, la flotte française et la flotte anglaise passeront entre les mains des Allemands et des Italiens et vous serez pris entre eux et les Japonais. Nous ferons de 
notre mieux pour vous éviter cette extrémité et nous ne désespérons nullement de réussir. Mais vous feriez sagement de prévoir le pire.

Bien amicalement,

Lefebvre

P. S. Seignobos habite en ce moment la Bretagne. Voici son adresse : Tachen-Bien, par Ploubazlanec (Côtes-du-Nord). Il sera très content si vous lui écrivez pour le remercier.

(1) Pierre Mille (1864-1941), romancier et journaliste au Temps.

(2) Charles-Victor Langlois (1863-1929), historien et archiviste français, spécialiste du Moyen Âge.

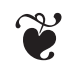

Boulogne-sur-Seine, 1e 11 octobre 1945

Cher Monsieur,

Non seulement j'ai gardé souvenir de vous, mais je me suis plusieurs fois demandé quel pouvait être votre sort au cours de ces années de tourmente. J'ai été content d'apprendre que vous êtes sain et sauf.À part quelques dommages causés à ma maison par les bombardements et qui sont peu de chose, par comparaison, la guerre ne m'a pas affecté personnellement, mais j'ai fait une perte cruelle en la personne de mon frère Théodore, professeur de géographie à la Faculté de lettres de Poitiers ${ }^{1}$, qui a été décapité à la hache à la prison de Wolfenbüttel près de Brunswick où il avait été emmené avec une trentaine d'habitants de Poitiers. Neuf autres ont été mis à mort avec lui. Ils avaient pris part dans la Résistance.

La Sorbonne a continué à enseigner tant bien que mal. J'ai été mis à la retraite le $1^{\text {er }}$ octobre 1941, ayant atteint l'âge de 67 ans. Néanmoins, mes collègues m'ont demandé et j'ai consenti à continuer comme auparavant à titre de chargé de cours complémentaires, afin de ne pas donner l'occasion à la trahison de mettre un de ses partisans à ma place. Mais j'ai refusé de continuer cette année 1945-46, à remplir ces fonctions à peu près gratuites. Je ne sais qui me succédera ${ }^{2}$.

Les Annales historiques de la Révolution [française] n'ont pu faire paraître qu'un seul numéro depuis le désastre : c'est le n 3 de 1940 ! Leur imprimeur me donne à espérer qu'il pourra faire paraître le n 4 de 1940 d'ici la fin de l'année. L'abonnement de 1940 qui reste fixé à 45 francs - ce 
qui n'est pas beaucoup aux prix d'aujourd'hui - n'a pas été payé par beaucoup d'abonnés, comme vous pourriez le penser, en sorte que nous ne savons pas sur quel nombre nous pouvons compter. Mais après le $\mathrm{n}^{\circ} 4$ et dernier de 1940, nous passerons au n 1 de 1945 et nous verrons l'effet.

Je ne pense pas que vous fussiez inscrit comme abonné en 1939? Je pense donc répondre à votre intention en vous inscrivant pour 1946 ? Si vous désirez recevoir les numéros de 1940, dites le moi. Bien que nous ne prenions pas de nouveaux abonnés au prix ancien, j'en fais mon affaire, en ce qui vous concerne.

J'ai lu avec intérêt votre article sur Rousseau ${ }^{3}$. Je crois pouvoir adhérer à ce que vous dites du culte littéraire et du culte politique de Jean-Jacques et vous avez recueilli à cet égard des précisions intéressantes. Mais il me semble que vous tendez à faire croire que l'influence politique de Rousseau a disparu avec son culte. Je ne le crois pas. J'ai trop bien conservé le souvenir de l'impression saisissante que j'ai ressentie en ouvrant le Contrat social. «L'homme est né libre, et partout il est dans les fers ! » et en rencontrant le fameux passage du Discours sur l'origine de l'inégalité. À cette impression, je ne puis comparer que celle du Manifeste communiste, dans sa première partie. Et l'influence de Rousseau sur les révolutionnaires me paraît considérable du moins en ce qui concerne le principe de la souveraineté. Et sur Robespierre donc ! La définition de la volonté générale qui a une base morale (la volonté du peuple n'est générale que lors qu'elle ne tient compte que du bien général et non de l'intérêt personnel des électeurs) est peut-être démodée, mais son inspiration morale reste bien celle d'une véritable démocratie.

Mais c'est assez discourir. Peut-être vous reverrais-je en France et pourrons-nous nous entretenir longuement de tout cela. En attendant, croyez à mes sentiments bien amicaux.

Lefebvre

(1) Sur Théodore Lefebvre, voir $A H R F$, t. 18, 1946, p. 283 et ibid., t. 19, 1947, p. 68-70.

(2) «Sur l'enseignement de M. G. Lefebvre en Sorbonne », AHRF, t. 18, 1946, p. 185.

(3) «The Cult of Rousseau and the French Revolution », Journal of the History of Ideas, t. 6, avril 1945, p. 197-212. 
Université de Paris

le 28 avril 1946

Faculté des lettres

Institut d'Histoire

de la Révolution Française

\section{Boulogne-sur-Seine \\ 86 , Bd Jaurès}

Cher Monsieur,

Votre envoi est arrivé à bon port. Ce fut une heureuse surprise, car depuis six ans, nous avions perdu le goût des friandises qu'il contenait, ou peu s'en faut. Ainsi soyez sûr qu'elles ont été appréciées hautement et veuillez bien accepter nos remerciements reconnaissants pour votre amicale attention.

Je voudrais bien, à mon tour, vous en donner un témoignage. Je ne pourrais guère vous envoyer que des livres français. Mais lesquels ? Si quelques-uns pouvaient vous être agréables, ou utiles, vous me ferez plaisir en mes les indiquant.

Vous avez dû maintenant recevoir les numéros 3 et 4 des Annales [historiques de la Révolution française] pour 1940. Le premier numéro de 1946 partira, je crois, après-demain. Je vous remercie d'avoir pris avis de satisfaire notre trésorier. Nous aurons une assemblée générale le 19 mai ${ }^{1}$. Nous tâcherons de mettre en train une table des Annales pour les années 1921-1940.

Bien amicalement vôtre

Lefebvre

(1) «Assemblée générale de la Société des études robespierristes », AHRF, t. 18, 1946, p. 96 . 
Université de Paris

Faculté des Lettres

17, rue de la Sorbonne

Paris, le 10 septembre 1946

\author{
Institut \\ d'histoire moderne \\ $\&$ contemporaine
}

Cher ami,

Je suis content de vous savoir rendu à vos études et d'apprendre que vous êtes installé dans un collège et une ville qui vous plaisent et d'où vous pourrez aller travailler à Chicago où vous trouverez notre ami [Louis] Gottschalk ${ }^{1}$. J'ai signalé votre nouvelle adresse à notre imprimeur. Le numéro 3 des Annales [historiques de la Révolution française] sera expédié au début d'octobre au plus tard. S'il ne vous parvenait pas, donnez m'en avis et je vous en expédierai un nouvel exemplaire. Vous y trouverez le compte-rendu de notre assemblée générale [du 19 mai 1946]. Le nombre des abonnés croît lentement : je ne couvre pas encore les frais mais nous avons une encaisse suffisante pour attendre des jours meilleurs.

Je vous renouvelle mes remerciements pour votre bienveillante attention. Je vais vous envoyer la Grande Peur de $1789^{2}$ qui est ce que je suis le plus content d'avoir écrit, et le volume sur Napoléon ${ }^{3}$ que je crois avoir bien réussi, du moins, j'espère que vous ne les possédez pas encore. Si vous en préfériez d'autres, dites le moi ; j'attendrai quelque temps avant de faire l'envoi parce [que] je n'ai pas de photographie de moi qui ne soit ancienne. Je vais me faire tirer une autre; d'ici là vous aurez le temps de me donner un avis si c'est nécessaire.

Je vous souhaite une bonne année d'enseignement et la reprise de quelque travail de recherche personnelle.

Bien amicalement vôtre

Lefebvre

(1) Louis Gottschalk (1899-1975), historien américain, spécialiste de la Révolution française.

(2) La Grande Peur de 1789, Paris, A. Colin, 1932, 272 p.

(3) Napoléon, Paris, Alcan, 1935, Collection «Peuples et civilisations », t. XIV, 606 p. ; deuxième édition, revue et corrigée, Paris, Presses universitaires de France, 1941, 606 p. 
Boulogne s/Seine, 6-4-[19]47

\section{Cher ami,}

Nous avons bien reçu votre colis. Ne doutez pas qu'il ait été accueilli avec plaisir, car la situation ici reste toujours fort médiocre. Je pense d'ailleurs que les professeurs des États-Unis rencontrent aussi des difficultés. Et vous ne devez pas être plus rassurés que nous sur la situation internationale.

Je suis content que mes envois de livres vous soient parvenus et qu'ils vous aient intéressés. [R. R.] Palmer ${ }^{1}$, de Princeton, a traduit mon Quatre-vingt-neuf et pense faire paraître sa version à la fin de cette année ${ }^{2}$. Beatrice Hyslop ${ }^{3}$ a offert de traduire mon Directoire.

J'ai traversé le rude hiver sans dommage mais j'ai été accaparé à tel point par les réunions de sociétés, les conférences et les articles de presse, sans parler des Annales [historiques de la Révolution française] dont la remise en train m'a pris beaucoup de temps, que j'ai dû interrompre la rédaction du volume de la Collection Halphen et Sagnac sur la Révolution ${ }^{4}$ qu'on m'a demandé de récrire seul cette fois, de fond en comble. Je vais partir pour Strasbourg où s'ouvre mardi le Congrès des Sociétés savantes et au retour j'espère que je vais pouvoir m'y remettre et consacrer tout l'été à cette entreprise.

Je souhaite que vous puissiez vous consacrer quelque peu à de nouvelles recherches. Mes vœux vous accompagneront: veuillez bien croire à mes sentiments fidèlement amicaux.

(1) R. R. PALmer (1909-2002), historien américain, auteur de plusieurs études sur la Révolution française.

(2) La traduction anglaise de Quatre-vingt-neuf, Paris, Maison du livre français, 1939, a été publiée en 1947 par Princeton University Press sous le titre The Coming of the French Revolution.

(3) Beatrice Hyslop (1899-1973), historienne américaine, spécialiste de la Révolution française, surtout des cahiers de doléances de 1789.

(4) La Révolution française, Paris, Alcan, 1930, en collaboration avec Philippe Sagnac et Raymond Guyot, Collection «Peuples et civilisations », t. XIII, 583 p. ; nouvelle rédaction, La Révolution française, Paris, Presses universitaires de France, 1951, 673 p. 
Mon cher ami,

Votre envoi nous est parvenu en bon état; il nous a rendu grand service et sa variété nous a été fort agréable. Mais je suis un peu inquiet en songeant au sacrifice qu'il vous a imposé car on rapporte que la situation des professeurs américains n'est pas aussi satisfaisante qu'ils la souhaiteraient. Ne vous croyez pas obligé de nous aider en vous imposant des privations.

Je n'espère pas vous revoir de sitôt en France malheureusement, mais j'espère que vous pouvez continuer à travailler. La difficulté doit être que peu de documents doivent se trouver à votre disposition.

La situation générale ne s'améliore pas, et s'en faut. Et la politique intérieure des États n'est pas plus consolante que la politique internationale. J'aurais pourtant bien aimé passer mes dernières années en paix.

Pour le moment, je suis attelé à la rédaction d'une nouvelle édition du volume relatif à la Révolution dans la collection Halphen et Sagnac. [R. R.] Palmer, de Princeton, a achevé la traduction de mon Quatre-vingtneuf; on l'imprimera à la fin de l'année. Je vous envoie un tiré à part d'une étude que j'ai publié dans la Revue historique'.

Adieu, mon cher ami. Que l'année vous soit légère.

Bien cordialement, 
Boulogne-sur-Seine, 25 juillet 1953

$\mathrm{B}^{\mathrm{d}}$ Jean Jaurès

Cher ami,

Miss Beatrice Hyslop m'a transmis la lettre collective que douze historiens américains, dont vous êtes, ont signé avec elle à l'occasion de mon entrée prochaine dans la quatre-vingtième année. Elle m'a touché plus que je ne saurais dire ${ }^{1}$.

Si ma vie se prolonge, j'espère ne pas décevoir votre vœu de voir ces années employées comme celles que l'ont précédées et retrouver le plaisir de vous voir ici et d'apprendre que vos travaux se poursuivent.

Miss Hyslop a joint le présent de mes collègues des États-Unis. Après les vacances, j'examinerai avec vos amis de France comment l'employer au profit des études qui nous sont chères.

Bien cordialement vôtre

Lefebvre

(1) Voir American Historical Review, t. 59, avril 1954, p. 819-820. 
Boulogne-sur-Seine, 28-12-[19]54

Cher collègue et ami,

J'ai été touché de votre attention. Je vous remercie de m'avoir envoyé vos vœux pour l'année qui va commencer et j'en forme pour vous de tout pareils.

Il m'est réconfortant de savoir que vous êtes satisfait de votre séjour à Fayetteville [Arkansas], et que vous y trouvez le moyen de travailler. J'espère donc entendre parler de vos publications futures.

[Louis] Gottschalk est à Paris avec sa femme et l'un de ses fils. J'ai pu ainsi lui renouveler mon témoignage de reconnaissante satisfaction pour l'honneur que m'a fait l'Association des historiens américains ${ }^{1}$. Il compte demeurer en Europe une grande partie de l'an qui va commencer ; jusqu'en septembre, je crois.

Bien cordialement,

Lefebvre

(1) Voir American Historical Review, t. 59, avril 1954, p. 813, 519-520. G. Lefebvre fut nommé membre honoraire de l'American Historical Association en décembre 1953. 